\title{
Review of Pembrolizumab and other Anti PD-1/PD-L1 Antibodies
}

\author{
Caleb P Gottlich MS* \\ Orthopedic Surgery Department, Johns Hopkins School of Medicine, USA
}

Submission: November 13, 2018; Published: December 21, 2018

*Corresponding author: Caleb P Gottlich MS, Orthopedic Surgery Department, Johns Hopkins Children's Center, Johns Hopkins School of Medicine, Baltimore, MD 21287, College of Medicine, Texas A\&M Health Science Center, Dallas, TX, 75246

\begin{abstract}
Immunotherapies are rapidly becoming favored amongst physicians for the treatment of various oncologic process due to their more favorable adverse reaction profile in addition to their increased ability to target specific cancer tissue as opposed to the usual widespread devastation of healthy tissue that can accompany chemotherapy and radiation therapy. One class of immunotherapies that has shown exquisite promise in terms of efficacy and limited immune related adverse events is the anti-PD-1/PD-L1 antibodies, which falls under the immunotherapy classification of check point inhibitor. In this paper, we will give a brief review of the background, mechanism, and side effects common to PD-L1/ PD-1 inhibitors as a cancer therapy.
\end{abstract}

Keywords: Anti PD-L1/PD-1; Pembrolizumab; Immunotherapy; Checkpoint inhibitor

\section{Background}

Immunotherapies are rapidly augmenting, sometimes even supplanting, the previously commonplace tactic of chemotherapy and/or radiation therapy in the treatment of several cancers. These treatment modalities are favored due to their heightened ability to specifically target cancer cells while simultaneously sparing healthy tissue that is usually considered to be standard collateral damage when using the less-specific treatments of chemotherapy or radiation. This can result in less severe side effects for the patients, while also reducing many of the complications attributable to less specific therapies.

In recent years the use of pembrolizumab, and other PD-1/ PD-L1 inhibitors, as a therapeutic agent for a variety of cancers has become more pronounced. Beginning in May of 2017, the FDA approved pembrolizumab for the treatment of non-small cell lung carcinoma after demonstrating an overall response rate (55\%) that was twice as effective as the previous standard of care therapy.1 In addition to the treatment of non-small cell lung cancer, anti PD-1/PD-L1 antibodies are also approved for the treatment of melanoma, bladder cancer, renal cell carcinoma, and Hodgkin lymphoma [1]. Several studies have gone on to validate the positive response rates seen in the initial studies of pembrolizumab in cases advanced melanoma as well as nonsmall cell lung carcinoma. Studies are also being conducted investigating the response rate that other cancers will show when treated with pembrolizumab in a series of trials called KEYNOTE clinical trials, which is still ongoing.

\section{Mechanism of Action}

One commonly found feature in cells of a tumor compared to cells of normal tissue is an aberrancy in the progress through the cell cycle. Generally, a mutation in the regulatory components of this intracellular process will experience a mutation resulting in either over-expression or under-expression of proteins that will allow for unregulated progression through the cell growth cycle.

The mechanism of action of pembrolizumab is that of a monoclonal antibody that acts to target against the PD-L1 protein. This inhibits said protein's interaction that normally occurs between the PD-1 protein expressed on the surface of host T-Cells [2]. Under normal circumstances the interaction of these two proteins, PD-1 and PD-L1, would result in an inhibitory cascade within the cells expressing PD-L1, halting an apoptotic process and effectively allowing these cells to "hide" from the host immune system; Pembrolizumab acts to inhibit this inhibitor which leads to the continuation of the apoptotic process.

One somewhat debated topic in the use of this therapy is the patient population that this drug is indicated for. An invaluable tool that has FDA support and is widely accepted in 
the treatment community is the use of the Tumor Proportion Score (TPS). This is calculated using immunohistochemical testing of the tumor specimen for relative expression of the PDL1 surface protein. If the sample shows greater than or equal to $50 \%$ of viable tumor cells displaying membrane staining of any intensity, this is a properly indicated situation likely to result in the desired response. A recent publication by Sunshine et. al. however summarized data from 1400 patients, which showed that demonstrable responses have been seen in $15 \%$ of patients who have been treated with anit-PD-1/PD-L1 therapies who have been considered PD-L1 negative on immunohistochemical testing [1].

\section{Side Effects}

Side effects of using this drug have been well documented, often presenting as fatigue, anorexia, dermatologic manifestations, endocrine derangements, pulmonary deficits or inflammatory changes of visceral organs such as liver or kidney [2-5]. The most common dermatologic and soft tissue manifestations consist of pruritic rash, hypopigmentation or myalgias with one report of eosinophilic fasciitis and vasculitis found in the literature [5-7].

Smoking is an important factor to consider regarding the likelihood of side effects associated with pembrolizumab therapy. Pembrolizumab, along with the other drugs in the anti PD-1/PDL1 monotherapy class, are known to have a less severe side effect profile in general when compared to standard therapies, but when side effects do arise are severe and can even be deadly. Naidoo et al recently published a paper looking at the incidence of pneumonitis, an uncommon but serious side effect seen after anti PD-1/PDL-1 treatment. Of these cases that were reported, the majority occurred in those patients who had recently stopped or were current smokers. Most cases have shown resolution with a hiatus in administration of the therapy [8-10].

Some cases of infusion reactions have been reported with the use of pembrolizumab, but most of the adverse reactions associated with the use of this therapy are thought to be immune mediated. Very few serious reactions have been reported, with as few as $5 \%$ considered high grade adverse events. Because this is still a relatively new drug under the early phases of investigation, however, more information is needed to fully understand the range of adverse events. There is no currently published definition on the optimal length of treatment, but it has been given up to two years. Across all published clinical trials, there is consistent reporting of the rates of adverse events showing very few mortalities and generally close to $10 \%$ severe (grades 3-5) adverse events [11,12].

\section{Conclusion}

Immunotherapies, such as check point inhibitors, offer a new and exciting prospect in the treatment of cancer. These therapies can impart more specificity in their ability to target tumorigenic tissues thereby sparing healthy tissues that are often damaged in traditional treatments. The PD-1/PD-L1 inhibitors are a relatively new class of checkpoint inhibitors that have shown great promise in clinical trials and early reports with a high efficacy and a low serious adverse event profile. Its novel mechanism of action has made it a great candidate for several types of cancers, namely non-small cell lung cancer as well as advanced melanoma. Generally, those tumors with a tumor proportion score of greater than $50 \%$ on immunohistochemistry show the best response. More investigation is needed to optimize treatment protocol as well as test the boundaries of its efficacy regarding unexplored cancer phenotypes.

\section{References}

1. Sunshine, J, Taube, JM (2015) PD-1/PD-L1 inhibitors. Current opinion in pharmacology 23: 32-38.

2. Center for Drug Evaluation and Research (2017) Approved Drugs Pembrolizumab (Pembrolizumab). U S Food and Drug Administration Home Page, USA.

3. An immunotherapy that may help fight your cancer (2018) it's tru. Pembrolizumab. An Immunotherapy that may help fight your cancer. it's tru. Pembrolizumab. Pembrolizumab ${ }^{\circledR}$ (pembrolizumab) | AntiPD-1 Immunotherapy, USA.

4. Ott PA, Bang Y, Berton-rigaud D (2017) Safety and Antitumor Activity of Pembrolizumab in Advanced Programmed Death Ligand 1 - Positive Endometrial Cancer: Results From the KEYNOTE-028 Study. J Clin Oncol 35(22): 2535-2541.

5. Patnaik A, Kang SP, Rasco D (2015) Phase I Study of Pembrolizumab (MK-3475; Anti - PD-1 Monoclonal Antibody) in Patients with Advanced Solid Tumors. Clin Cancer Res 21(19): 4286-4293.

6. Wang PF, Chen Y, Song SY (2017) Immune-related adverse events associated with anti-PD-1/PD-L1 treatment for malignancies: A metaanalysis. Front Pharmacol 8: 730

7. Khoja L, Maurice C, Chappell M (2016) Eosinophilic Fasciitis and acute Encephalopathy Toxicity from Pembrolizumab. Eur J Cancer 54(3): 172-178.

8. Baxi S, Yang A, Gennarelli RL (2018) Immune-related adverse events for anti-PD-1 and anti-PD-L1 drugs: systematic review and metaanalysis 1:2-8.

9. Naidoo J, Wang X, Woo KM (2017) Pneumonitis in patients treated with anti-programmed death-1/programmed death ligand 1 therapy. J Clin Oncol 35(7): 709-717.

10. Naidoo J, Schindler K, Querfeld C (2017) Autoimmune Bullous Skin Disorders with Immune Checkpoint Inhibitors Targeting PD-1 and PDL1. Cancer Immunol Res 4(5): 383-389.

11. Naidoo J, Cappelli LC, Forde PM (2017) Inflammatory Arthritis: A Newly Recognized Adverse Event of Immune Checkpoint Blockade. Oncologist 22(6): 627-630.

12. Kwok, Yau, Chiu, Tse, Kwong (2016) Pembrolizumab (Keytruda). Human vaccines \& Immunotherapeutics 12(11): 2777-2789. 

CC (1) This work is licensed under Creative Commons Attribution 4.0 Licensz
DOI: 10.19080/GJPPS.2018.06.555696

\section{Your next submission with Juniper Publishers will reach you the below assets}

- Quality Editorial service

- Swift Peer Review

- Reprints availability

- E-prints Service

- Manuscript Podcast for convenient understanding

- Global attainment for your research

- Manuscript accessibility in different formats ( Pdf, E-pub, Full Text, Audio)

- Unceasing customer service

Track the below URL for one-step submission https://juniperpublishers.com/online-submission.php 